spores) description of aleuriospores (misspelled aleurospore) tends to confuse and complicate unnecessarily and would have been better covered in a short footnote. Individual treatment of the morphologically similar Microsporum gypseum and $M$. fulvum results in considerable repetition and gives undue importance to the need for separation of these two species. In contrast, the recognition of varieties, especially var. interdigitale, of Trichophyton mentagrophytes is epidemiologically of value and the information on these could be expanded and emphasised to advantage. Perhaps the most serious criticism is that there is no reference to the non-dermatophytes that cause disease clinically indistinguishable from ringworm and which are bound to be encountered. A short section on these fungi and the related issue of the use of cycloheximide, which is far from being absolutely necessary in isolation media, would have been useful.

These few criticisms in no way detract from the value of this booklet which will surely find a place on the bench of most routine microbiological laboratories.

J. C. Gentles

\title{
Basic medical microbiology
}

By R. F. Boyd and B. G. Hoerl. 1981. Little, Brown and Co., Boston. Pp. $x$ and 766. US\$ 22.00 .

This is the second edition of a textbook first published in 1977 and the authors have taken the opportunity to update the information in many of the chapters.

The book is arranged in nine major sections. The first section (nine chapters) is basic microbiology, mainly bacteriology, which lays the foundation on which the specifically medical parts are based. The subjects covered include a short historical introduction, bacterial cytology, nutrition, growth and reproduction, principles of cultivation, isolation and identification, enzymes, energy metabolism, metabolic control and genetics. A brief description of genetic engineering is included. The chapters are of necessity somewhat superficial but they are adequate for the purposes of the book, which then proceeds very logically through sections on the control of micro-organisms, host-parasite interaction and immunology to sections dealing with bacterial, viral and fungal diseases. Eleven chapters on bacterial diseases are organised under the headings of the bacterial types, i.e., the gram-positive cocci, the gram-negative enteric bacilli, etc., and in each case there are numerous subdivisions for topics such as classification, toxins and enzymes, antibiotic resistance, pathogenicity, laboratory diagnosis, epidemiology, treatment and control. The virology section (two chapters) includes the general biology of viruses and a survey of selected viral diseases. Fungal diseases are given only one chapter although their importance is not denied. Another chapter is devoted to medical parasitology and describes diseases produced by protozoa and the non-microbiological helminths and arthropods. The final part of the book consists of fairly detailed accounts of two topics of special interest, oral microbiology and nosocomial infections.

Each chapter, there are 34 in all, has a good selection of references and there is a detailed glossary. The book is well organised, quite well illustrated-some colour in the illustrations would be advantageous but would doubtless push up the present reasonable price-clearly written and can be recommended for undergraduates or others requiring a broad coverage of the medical aspects of microbiology.

D. G. SMITH

\section{Systemic effects of HBsAg immune complexes}

Edited by E. Bartoli, L. Chiandussi and S. Sherlock. 1981. Piccin Medical Books, Padua, Italy. Pp. xi and 308. $£ 21.75$.

This book is a bound reproduction, by poor-quality photocopying, of papers, and sometimes only summaries or abstracts, delivered during an international symposium held in Italy in September 1979. There is thus an irritating lack of consistency in the typesetting, quality of 